Journal of Clinical and Nursing Research

Research Article

\title{
Effects of Crown Lengthening on Bi, PLI and PD Levels in Patients with Different Periodontal Biotypes
}

Lu Bai

Department of Stomatology, Chifeng University Affiliated Hospital, Chifeng 024000, Inner Mongolia, China

[Abstract] Objective: To investigate the effect of crown lengthening on patients with different periodontal biological types. Methods: 60 patients with different periodontal biotypes treated in our hospital from May 2017 to February 2019 were retrospectively analyzed. According to the different periodontal biotypes, the above-mentioned patients were further divided into control group (thin fan type, 30 cases) and observation group (thick flat type, 30 cases). The periodontal probing depth (PD), plaque index (PLI), bleeding index (BI) and gingival margin position at 1 week, 1 month, 2 months and 3 months after operation were compared between the two groups. Results: The PD, Bi and PLI values of the two groups after treatment were lower than those before treatment $(\mathrm{P}<0.05)$; There was no significant difference in gingival margin position between the two groups after 1 week to 1 month and 1 month to 2 months $(\mathrm{P}>0.05)$;After 2 months to 3 months of treatment, the gingival margin distance of the observation group was larger than that of the control group, and the difference was statistically significant $(\mathrm{P}<0.05)$. Conclusion: Crown lengthening can effectively improve the periodontal indexes of different periodontal biotypes, and the gingival margin of thick and flat type patients moves more toward the crown.

Key words: Periodontal biotype; Crown lengthening; Periodontal index

Publication date: September, 2020

Publication online: 30 September, 2020

*Corresponding author: Lu Bai, 11118227@163.com

Periodontal biotype refers to the characteristics of periodontal soft tissue and alveolar bone tissue, which reflects the thickness of gingiva, the width of keratinized gingiva, the shape of crown and the height of gingival papilla, and is related to the quantity and shape of the lower alveolar ridge. It can be divided into thin fan type and thick flat type $\mathrm{e}^{[1-2]}$. Among them, the thin fan type bone is usually scallop shaped, with narrow and long gingival papilla and shallow crown neck protrusion. Permanent gingival retraction is easy to occur after inflammatory reaction, while thick and flat bone tissue is thicker, gingival papilla is wide and flat, crown neck convexity is obvious, gingival is not easy to atrophy, but deep pocket and bone defects are easy to form after inflammatory reaction ${ }^{[3-4]}$. At present, crown lengthening is often used for symptomatic treatment in clinic, but the curative effect of different periodontal biological types is different. Based on this, the purpose of this study was to investigate the effect of crown lengthening on different periodontal biotypes. The research situation is as follows.

\section{Material and methods}

\subsection{The general information}

A retrospective analysis was conducted on 60 patients with different periodontal biotypes treated in our hospital from May 2017 to February 2019. According to the different periodontal biotypes, the above selected patients were further divided into control group (thin fan type, 30 cases) and observation group (thick flat type, 30 cases). There were 18 males and 12 females in the control group. The age range was 18-49 years old with an average age of $(33.51 \pm 2.23)$ years old. There were 13 bicuspid teeth, 10 upper central incisors and 7 first molars. There were 17 males and 13 females in the observation group. The age range was 18-50 years old, with an average age of $(33.68 \pm 2.31)$ years. There were 
12 bicuspid teeth, 10 upper central incisors and 8 first molars. There was no significant difference in general data between the two groups $(\mathrm{P}>0.05)$.

\subsection{Inclusion criteria and Exclusion criteria}

(1) Inclusion criteria: The teeth with broken ends less than $3 \mathrm{~mm}$ in subgingival area have the value of preservation; Patients with complete medical records and follow-up data; There were no contraindications. (2) Exclusion criteria: Pregnant and lactating women; Coagulation dysfunction; Patients with systemic diseases; In recent 3 months, the drugs that can cause periodontal tissue hyperplasia were taken; Expression disorder or mental illness; Patients with severe infectious diseases.

\subsection{Methods}

The gingival inflammation of the two groups was eliminated before surgery, oral hygiene was paid attention to, local anesthesia was performed, the location and scope of the broken teeth were verified, the incision location was determined, and the gingival margin was coordinated with the adjacent teeth after surgery. The incision can be extended to 1-2 healthy teeth with 15C blade. Open the full-thickness flap, remove the residual gingival tissue, scrape away the granulation, fully expose the tooth cross-section. Osteoplasty and osteotomy were performed with ultrasonic osteotome (Guilin woodpecker Medical Equipment Co., Ltd., ULTRASURGERY), and part of the alveolar bone was removed, so that the broken end was away from the alveolar ridge. The apical distance should be at least $3 \mathrm{~mm}$. Meanwhile, it should move to the top of the bone ridge of the adjacent teeth to meet the biological width. The residual periodontal ligament fiber on the root surface should be removed to prevent postoperative recurrence. The flap should be reset and attached to the root surface for observation. In the control group, gingival tear was avoided, gingival retraction and bone resorption were prevented, and bone mass was reduced timely. For the thick flat teeth in the observation group, the amount of gingival tissue and bone tissue removal was increased, the shape and thickness of gingival flap were trimmed, the gingival flap was reset to the top of alveolar ridge, and the fit of the flap was checked. Sterile gauze was used to compress hemostasis, and " 8 " suture was used, and periodontal protectant was placed. After operation, fangchlorhexidine gargle was given (Jilin Lisheng Pharmaceutical Co., LTD., National drug approval number H20003445), 10-20 ml/time, gargle for at least
2-5 min in the mouth, gargle for 2-3 weeks, timely ice cold compress, strengthen oral hygiene, suture can be removed 10 days after surgery, and porcelain fused to metal crown restoration can be carried out 3 months after operation.

\subsection{Evaluation Index}

The probing depth (PD) of 6 sites in each tooth was detected by Florida periodontal electron probe, the bleeding index (BI) was detected by Williams periodontal probe, and the plaque index (PLI) was measured by loe and siline plaque index 3 months after operation; The position of gingival margin was recorded 1 week to 1 month, 1 month to 2 months, 2 months to 3 months after operation. Vernier caliper was used to measure the distance from the apex of gingival margin to the cutting end. If the gingival root retracted, the record was negative, if the gingival crown shifted, it was positive.

\subsection{Statistical methods}

SPSS 12.0 software was used for statistical analysis. The measurement data were expressed as $(\mathrm{x} \pm \mathrm{s}), \mathrm{t}$ test was used for comparison, and $\chi^{2}$ test was used for counting data. $\mathrm{P}<0.05$ is considered as statistically significant difference.

\section{Results}

\subsection{Periodontal index}

PD, BI and PLI values of the two groups after treatment were all lower than those before treatment, with statistically significant differences $(\mathrm{P}<0.05)$. Shown in Table 1 .

Table 1. Comparison of periodontal indexes between the two groups $(\mathrm{x} \pm \mathrm{s})$

\begin{tabular}{ccccc}
\hline Group & time & PD $(\mathrm{mm})$ & BI & PLI \\
\hline & $\begin{array}{c}\text { Before } \\
\text { treatment }\end{array}$ & $3.36 \pm 0.75$ & $1.29 \pm 0.62$ & $0.92 \pm 0.37$ \\
$\begin{array}{c}\text { Control group } \\
(\mathrm{n}=30)\end{array}$ & After treatment & $2.67 \pm 0.58$ & $0.75 \pm 0.34$ & $0.48 \pm 0.35$ \\
& $t$ & 3.986 & 4.183 & 4.732 \\
& $P$ & 0.000 & 0.000 & 0.000 \\
& $\begin{array}{c}\text { Before } \\
\text { treatment }\end{array}$ & $3.34 \pm 0.72$ & $1.27 \pm 0.58$ & $0.90 \pm 0.36$ \\
Abservation & After treatment & $2.42 \pm 0.69$ & $0.29 \pm 0.53$ & $0.46 \pm 0.39$ \\
group (n=30) & $t$ & 5.053 & 6.832 & 4.541 \\
& & & & \\
& $P$ & 0.000 & 0.000 & 0.000 \\
\hline
\end{tabular}




\subsection{Position of gingival margin}

Comparison of gingival margin positions between the two groups after treatment for 1 week to 1 month and 1 month to 2 months showed no statistically significant difference ( $\mathrm{P}>0.05)$.After 2 to 3 months of treatment, the distance of gingival margin in the observation group was greater than that in the control group, with statistically significant difference $(\mathrm{P}<0.05)$. Shown in Table 2.

Table 2. Comparison of changes of gingival margin position between the two groups $(\mathrm{x} \pm \mathrm{s}, \mathrm{mm})$

\begin{tabular}{|c|c|c|c|}
\hline Group & 1 week to 1 month & 1 to 2 months & 2 to 3 months \\
\hline Control group $(\mathrm{n}=30)$ & $-0.94 \pm 1.01$ & $0.11 \pm 0.49$ & $0.41 \pm 0.32$ \\
\hline Observation group $(\mathrm{n}=30)$ & $-0.91 \pm 1.09$ & $0.32 \pm 0.88$ & $0.96 \pm 0.91$ \\
\hline$t$ & 0.111 & 1.142 & 3.123 \\
\hline$P$ & 0.912 & 0.258 & 0.003 \\
\hline
\end{tabular}

\section{Discussion}

Gingival biotype can be divided into thin fan type and thick flat type according to tissue morphology, of which thick flat type accounts for $85 \%$ and thin fan type accounts for $15 \%{ }^{[5]}$. Clinical results show that the thick flat type gingiva is thick, with more fibers, more attached gingiva, and thicker alveolar bone, which is prone to inflammatory reaction after adverse stimulation, leading to gingival hyperplasia or root displacement of the combined epithelium, and then leading to the formation of periodontal pocket ${ }^{[6]}$. The thin fan type gingiva and alveolar bone undulate in the adjacent face and labiobuccal surface have obvious difference. The alveolar bone is thin, and the labiobuccal surface is prone to bone fracture and fenestration, with less attached gingiva. After excessive stimulation, it is easy to cause gingival retraction of adjacent face and labiobuccal surface, and gingival retraction is also easy to occur during crown restoration, which leads to gray line of gingival margin and black triangle between teeth, which seriously affects the aesthetics ${ }^{[7-8]}$. Therefore, it is of great significance to choose an effective treatment for different periodontal biotypes.

According to the clinical characteristics of thin fan type and thick flat type teeth, crown lengthening was selected for treatment. The results showed that the PD, $\mathrm{Bi}$ and PLI values of the two groups after treatment were lower than those before treatment, but after 2 months to 3 months of treatment, the crown growth of thick flat gingiva in the observation group was more significant. The reason is that the crown lengthening surgery used in this study is a kind of oral surgical treatment, that is, the redundant gingival tissue and bone around the upper teeth are removed through the surgical method, so as to reduce the position of gingival margin, expose the healthy tooth tissue, and lengthen the clinical crown, so as to facilitate the next step of tooth repair and solve the aesthetic problem of gingival morphology ${ }^{[9]}$. In the process of operation, the incision position should be determined first, and the position of gingival margin should be coordinated with the adjacent teeth. Flap and curettage should be carried out to fully expose the tooth cross-section, so as to facilitate the repair of alveolar bone, and complete root surface leveling to prevent postoperative recurrence. Then, the gingival flap was trimmed, reset and sutured, and periodontal plug was placed at last ${ }^{[10]}$. For the thin fan shaped teeth with thin alveolar bone and narrow attached gingiva, the gingiva and bone tissue should not be excessively trimmed to prevent gingival recession. For the thick flat teeth with thick and tough gums and wide attached gingiva, the amount of gingival and bone tissue removal should be increased during crown lengthening to prevent postoperative hyperplasia. In addition, this study showed that the periodontal index and gingival margin position of the teeth with different periodontal biotypes were relatively stable at 3 months after operation. Therefore, full crown restoration is suitable for 3 months after operation. At the same time, pay attention to oral health maintenance after operation, which is more conducive to reduce plaque index. It can be seen that crown lengthening can effectively treat the teeth with different periodontal biological types, but we should pay attention to the clinical characteristics of different types of patients in the process of operation.

To sum up, crown lengthening can effectively improve the periodontal indexes of patients with different periodontal biological types. However, the effect of thick and flat type teeth is more significant, and the gingival margin moves more toward the crown after operation, which creates conditions for making reasonable restoration, which is worthy of clinical 


\section{application.}

\section{Reference}

[1] Li L. Influence of different periodontal biotypes on the treatment effect of crown lengthening[J]. Hainan Medical Journal, 2018, 29(8): 1169-1171.

[2] Ma N, Yu C, Liu HP. Effect of periodontal biotype on red and white aesthetic effect of simultaneous guided bone regeneration in aesthetic area $[\mathrm{J}]$. Chinese Journal of aesthetic medicine, 2019, 28(1): 138-141

[3] Yan XD, Zhang LN, Ren XY. Research progress of gingival biotype in anterior teeth area[J]. Stomatological Research, 2019, 35(2): 116-118.

[4] Shen T, Xu JP, Zhou DS. Effects of different gingival biotypes on aesthetic restoration of all ceramic crown of upper anterior teeth [J]. Chinese Journal of aesthetic medicine, 2018, 27(11): 82-85.

[5] Zhao YX, Zhou Y, Pu LI, et al. Analysis of related factors of gingival biotype of maxillary central incisors[J]. Stomatology,
2017, 37(7): 615-620

[6] Li Y, Hu JT, Peng SJ, et al. Clinical value of orthodontic treatment on alveolar bone mineral density of different periodontal biotypes[J]. Journal of Kunming Medical University, 2017, 38(9): 113-118.

[7] Zhu YQ, Chen LJ, Lv J, et al. Application of ultrasonic osteotome in thick gingival biological anterior crown lengthening[J]. Journal of dental pulp Periodontology, 2014, 24(3): 159-161.

[8] Liu XJ, Chen W, Xiao R. Effects of different restoration materials on periodontal status and prognosis after anterior crown lengthening $[\mathrm{J}]$. Journal of oral and maxillofacial prosthodontics, 2019, 20(3): 134-137.

[9] Hao XH, Zhang XR, Liu BY. Crown lengthening combined with fiber post in the repair of severe defects of anterior teeth and premolars[J]. Chinese Journal of medicine and clinic, 2019, 19(12): 2066-2068.

[10] Mo ZY, Gao YB. Effect of modified crown lengthening and orthodontic traction plus crown lengthening in the treatment of upper anterior gingival body defect $[\mathrm{J}]$. Chinese medical guide, 2019, 16 (24): 115-118. 